\title{
IEEJ Journal of Industry Applications
}

\section{《 EDITORIAL BOARD 》}

EDITOR IN CHIEF KIYOSHI OHISHI (Nagaoka Univ. of Tech.)

《POWER Electronics ANd Its APplications (D6) 》

Co-Editor In ChIEF Hideaki FuJita (Tokyo Inst. of Tech.)

AsSOCIATE EDITOR TOMOKI YokоYAMA (Tokyo Denki Univ.)

Hitoshi HaGa (Nagaoka Univ. of Tech)

NOBUKAZU Hoshi (Tokyo Univ. of Science)

TADASHI Suetsugu (Fukuoka Univ.)

SHIN-ICHI HAMASAKI (Nagasaki Univ.)

《Motion Control, RoBotics, SENSING AND THEIR APPLICATIONS (D7) 》

CO-EDITOR IN CHIEF YASUTAKa Fujimoto (Yokohama National Univ.)

ASSOCIATE Editor MasaAki Shibata (Seikei Univ.) Jun Ishikawa (Tokyo Denki Univ.)

Tadanao Zanma (Chiba Univ.) Hiroshi Fujimoto (The Univ. of Tokyo)

《 Electric Machine, Motor Drive AND THEIR APPLiCATIONS (D8) 》

CO-EDITOR IN CHIEF TAKASHI KosAKA (Nagoya Inst. of Tech.)

Associate Editor Hisao Kubota (Meiji Univ.) Keilchiro Kondo (Waseda Univ.)

Masayuki Sanada (Osaka Prefecture Univ.) AKio Toba (Fuji Electric)

\section{《INTERNATIONAL EDITORIAL ADVISORY BOARD》}

JiN-Woo AHN (Kyngsug Univ.)

Nicola Bianchi (Univ. di Padova)

Aldo Bogleteti (Politecnico di Torino)

TOKIO MarTin Buss (Technische Univ. München)

JAEHO CHOI (Chungbuk National Univ.)

Po-Tai Cheng (National Tsing Hua Univ.)

Bo Hyung Cho (Seoul National Univ.)

RIK W. DE DONCKER (RWTH Aachen Univ.)

Leopoldo G. Franquelo (Univ. de Sevilla)

GRAHAME HOLMES (RMIT Univ.)

JoHN Hung (Auburn Univ.)

Thomas M. JAHNS (Univ. of Wisconsin Madison)

JOHANN WALTER KOLAR (ETH Zurich)

Tsorng JuU (PeTer) Liang ( $N$. Cheng Kung $U$.)

ROBERT D LORENZ (Univ. of Wisconsin Madison)

KIM F. Man (City Univ. of Hong Kong)

Kouki MatsuSe (Meiji Univ.)

Roberto ObOe (Univ. di Padova)

Fang Z. Peng (Michigan State Univ.)

SEUNG KI Sul (Soul National Univ.)

VALERIY VYATKIN (Lulea Univ. of Tech.)

DEHONG XU (Zheijiang Univ.)

ZI-QIANG ZHU (The Univ. of Sheffield)
Hirofumi AKAGi (Tokyo Inst. of Tech.)

FREDE BLAABJERG (Aalborg Univ.)

DUSHAN BOROYEVICH (Virginia Polytechnic Institute and State Univ.)

CHANDAN CHAKRABORTY (Indian Institute of Tech. Kharagpur)

YAOW-Ming CHEN (National Taiwan Univ.)

MING CHENG (Southeast Univ.)

Mo-Yuen CHOW (North Carolina State Univ.)

J.A. FERREIRA (Delft Univ. of Tech.)

ThOMAS G HabeTLER (Georgia Tech)

YOICHI HORI (The Univ. of Tokyo)

YOUSEF IBRAHIM (Federation Univ. Australia)

Atsuo KaWAMura (Yokohama National Univ.)

PeTER Korondi (Budapest Univ. of Tech. and Economics)

JinJUN LiU (Xi'an Jiaotong Univ.)

REN C. LUO (National Taiwan Univ.)

NobUyUKi MATSUi (Nagoya Inst. of Tech.)

KWANG HeE NAM (Pohang Univ. of Science and Tech.)

KOUHEI OHNISHI (Keio Univ.)

ALFred RUfer $(E P F L)$

MaSAyOShi TOMizUKa (Univ. of California, Berkeley)

BOGDAN M. WILAMOWSKI (Auburn Univ.)

Peter Xu (Univ. of Auckland)

\section{《BOARD OF IEEJ INDUSTRY APPLICATIONS SOCIETY 》}

PRESIDENT

EDITOR IN CHIEF (JAPANESE JOURNAL)

EDITOR IN CHIEF (ENGLISH JOURNAL)

VICE PRESIDENTS

OFFICERS, PLANNING \& GENERAL AFFAIRS

OFFICERS, TREASURERS

OFFICERS, EDITORIAL AFFAIRS

OFFICERS, R\&D MANAGEMENT

OFFICERS, INTERNATIONAL AFFAIRS
NORIKO KAWAKAMI (TMEIC)

TOSHIYUKI MURAKAMI (Keio Univ.)

KIYOSHI OHISHI (Nagaoka Univ. of Tech.)

MASAAKI SHibata (Seikei Univ.)

RYOJI MIZUTANI (Furukawa Electric)

TAKASHI KOSAKA (Nagoya Inst. of Tech.)

Hideaki Minakata (Chiba Inst. of Tech.)

YUJI ENOMOTO (Hitachi)

Hitoshi HaYASHIYA (JR East)

JUN-ICHI ITOH (Nagaoka Univ. of Tech.)

HIROSHI FUJIMOTO (The Univ. of Tokyo)

TAKAHIRO URAKABE (Mitsubishi Electric)

SHINZO TAMAI (TMEIC)

KAN AKATSU (Shibaura Inst. of Tech.)
AUDITORS

OFFICERS
YASUShi MATSUMOTO (Fuji Electric)

YOSHITAKA IWAJI (Hitachi)

MaSaYUKi SANAda (Osaka Prefecture Univ.)

YUSHI MiUra (Osaka Univ.)

NobUKAZU Hoshi (Tokyo Univ. of Science)

AKIO TOBA (Fuji Electric)

ToshIHISA SHIMIZU (Tokyo Metropolitan Univ.)
KEIICHI HIROSE (NTT Facilities)

MASAHIKo TSUKAKOSHI (TMEIC)

YASUTAKA FUJIMOTO (Yokohama National Univ.)

MASAAKI KAIZUKA (Honda $R \& D$ )

YASUE MitsukURA (Keio Univ.)

KEIICHIRO KONDO (Waseda Univ.) 